\title{
A Literature Review of Different Criterias for Selecting and Cementing a post- $A$ review.
}

\author{
Partapjot Singh Grewal ${ }^{1}$, Kanu Priya Grewal ${ }^{2}$, Deepak Grover ${ }^{3}$ \\ ${ }^{1}$ M.D.S., Senior Lecturer, Department of Prosthodontics, BRS Dental college and Hospital,Sultanpur(Panchkula). \\ ${ }^{2}$ M.D.S., Senior Lecturer, Department of Pedodontics, National Dental College and Hospital, Gulabgarh , Dera Bassi. \\ ${ }^{3}$ M.D.S., Senior Lecturer, Department of Periodontics, National Dental College and Hospital, Gulabgarh, Dera Bassi.
}

Correspondence:

Department of Prosthodontics,

BRS Dental College and Hospital,

Sultanpur, Distt- Panchkula

(Haryana) , Pin-134118, INDIA

E-mail: partapjot_grewal@rediffmail.com

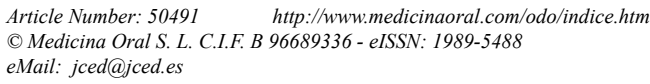

\begin{abstract}
The primary function of a corono-radicular post is to provide retention for a core, which replaces lost coronal tooth structure and retains the final restoration without compromising the apical seal of the endodontic filling. Therefore, it is important to select a post system that provides maximum retention, yet removes as little as possible of the remaining subgingival tooth structure. Several new esthetic dowel systems are available for the restoration of endodontically treated teeth, but little is known about how effectively these dowels seal the restored teeth. These post and core restorations are subjected to repeated tension, compression and torquing forces. Most forces clinically manifest themselves as tensile forces or shear stress on the post-cement-dentin interfaces. Recently, various endodontic dowel systems have been made available for restoring endodontic treated or mutilated teeth, making the choice difficult for the practitioner. In light of this, the current paper provides an insight into the various post and core systems available, the criteria and methods for their selection and cementation.
\end{abstract}

Key words: Esthetics dowel. Dowel systems. Cementing posts. Retention posts 


\section{Introduction}

Numerous types of dowels have been devised. Alloys such as stainless steel, platinum-

iridium, gold, and nickel-cobalt-chromium and latest materials like glass fiber posts, zirconia etc have been used. There has also been considerable variation in configuration of dowels, for example, parallel-sided, tapered, threaded, and smooth. The retention of various post systems, and the variables reported to affect retention include length, diameter and design of the post, canal shape and preparation, luting medium, method of cementation and location in the dental arch. All of the various aspects for selecting and cementing a post are discussed in this article.

\section{LENGTH}

As length of the post increases so does its retention. A post that is too short is bound to fail. Inadequate length of post is the leading cause of failure of restorations on endodontically treated teeth. Ideally, the post should be as long as possible without jeopardizing the apical seal and integrity of remaining root structure as stated by Stockton $L W$ (1) and Kurer et al (2). However if a post is too long, it may damage the seal of root canal or risk perforation .

\section{DIAMETER}

The increase in post diameter does not significantly effect its retention as stated by Standlee et al (3) and Kurer et al (2). It is because of the variations in canal morphology, such that an increase in surface contact between the post and canal walls does not occur proportionately with increased post diameter. The diameter of post should not be increased at the expense of the dentin, as the dentin provides a solid base required for the restoration of a tooth and the structural strength of a tooth depends on the quantity and inherent strength of the dentin.

3.DESIGN

A parallel-sided post is more retentive followed by the parallel tapered combination design, whereas tapered post is the least retentive as stated by Johnson et al (4). The lower retention obtained with the tapered-end post is attributed to the lack of parallelism in the apical portions. However a good adaptation is paramount. A well adapted, passively cemented, parallel post is considered most retentive with least stress. On the basis of retention and stress distribution, parallel-sided posts should be favoured for clinical use over parallel posts with tapered apical ends.

\section{SURFACE CONFIGURATION}

Surface configuration plays an important role in retention. The posts with the rough surfaces produce a higher strength of the bond to the cement than do the untreated posts, because of increase in surface area for bonding. The threaded posts are more retentive than the serrated posts, whereas the smooth posts have the least retention. The retentive values for sandblasted round parallel-si- ded dowels are significantly higher than the values of non sandblasted dowels of similar forms as stated by Balbosh A et al (5) and Nergiz et al (6).

$D$ 'Arcangelo $C$ et al (7) has shown by treating the surface of the fiber posts with silanization, acid etching with hydrofluoric acid and sand blasting through SEM analysis that presense of microretentive morphological changes, which certainly increase post-retentive properties without decreasing their flexural properties.

Monticelli $F(8)$ has shown the adverse effects of using Hydrofluoric acid for etching the fiber posts as it can cause the extensive damage to the fiber posts by giving rise to the micro-cracks and longitudinal fractures of the fiber layers.

\section{ADAPTATION OF THE POST}

A well adapted post has a better retention within the root canal. As studied by Chan FW (9) the resin adhesive in the well fitting canals provided very good retention. The well adapted post will resist torsion forces that will help indirectly in better retention.

\section{POST SPACE PREPARATION}

The mechanical removal of the sealer-impregnated dentin from the canal walls during post-space preparation is a critical step in achieving optimum post retention when res in cement is used. When sealers are introduced into the canal and obturation forces are applied, it is likely that sealer constituents penetrate into the dentinal tubules.

Therefore, when using resin cements a critical factor is the post-space preparation of the walls of the canal so that a "freshened" surface is produced into which resin cement could adequately flow and bond. Mayhew JT (10) showed that using a resin based sealer improves post retention.

\section{CHELATING AGENT}

In order to enhance retention, it is advised that before the cementation of a post, the canal space should be cleaned by application of a chelating agent to remove the smear layer. When smear layer is removed, it allows the cement to enter the dentinal tubules and provide micromechanical retention.

Commonly used agents for post space irrigation include EDTA or $5-5.25 \% \mathrm{NaOCl}$ that denatures protein and removes collagen, and Acids (such as $50 \%$ citric acid and $37 \%$ phosphoric acid) that remove the smear layer and demineralise dentin as stated by Keles A et al (11). Alcohol (ethanol) may be chosen as post space irrigant, because eugenol is fully soluble in alcohol, while only sparingly soluble in water. In addition, alcohol spreads readily over the entire dentinal surface because of its excellent wetting properties.

\section{LUBRICANTS}

While making custom post patterns with acrylic resin or wax, some type of canal lubricants are recommended in order to prevent adhesion of the pattern material to den- 
tin. However, if the lubricant remains within the canal at the time of final cementation it affects retention of the post, especially in the tapered post. Improved retention is reported in canals that are not lubricated. If a lubricant is needed it should be thoroughly removed before post cementation .

\section{TYPE OF CEMENT}

All posts, to a greater or lesser extent, gain their final retention by cementation into the tapered root canal. One criterion for selection of the cementing medium would be that the one chosen has the greatest retention. Many dental cements do not exhibit any real adhesion to the dentine or enamel surfaces. Their retention is essentially a locking of thin cement layer into irregularities of both the post and the canal.

The ability of a cement to retain a post influences the prognosis of the restoration. Cement that yields high retentive values will allow the use of shorter posts, thus maintaining the apical seal and preserving sound root structure. Various types of cements are used for post retention.

Several studies have shown no correlation between dowel retention and type of luting agent when conventional cements with different posts were used. However, Assif D and Ferber A (12) showed that when resin-based cements were used, a significant increase in dowel retention was shown compared with conventional cements. Adhesive resin cements are being used for cementation of the post because of their stronger bond to tooth structure. Current dentin bonding agents have hydrophilic and hydrophobic components that react with both the tooth structure and the resin. These agents achieve chemical adhesion as well as micromechanical bonding to dentin collagen and tubules, leading to reduced microleakage between the tooth and the restorations, thus reducing recurrent caries around restorations and minimizing coronal and apical contamination of the root canal system . 10. SILANE COUPLING AGENT APPLICATION The research hypothesis states that various surface treatments of glass fiber posts effects the tensile bond strength of resin cements. Silane coupling agents can achieve chemical bond with $\mathrm{OH}$-covered inorganic substance such as glass. Chemical bond between the resin cement and fiber posts is expected to contribute to the retention by coupling the post and resin materials by silane. The mechanism involved in enhancing the bond is the improvement in surface wettability of post after silane treatment as stated by Monticelli F et al (13). Silane with its low viscosity would assist substrate wetting and intimate contact between post-resin is established. Then the Van Der Walls forces become effective, providing the physical adhesion, which contributes the chemical reactions.

\section{BONDING AGENT}

Although the results obtained using different bonding agents can be variable, the use of different bonding agents do not result in any significant difference in retention.

\section{CEMENT APPLICATION}

Turner $\mathrm{CH}$ (14) suggested that posts should be cemented with cement placed into the canal space and also by coating the post prior to insertion. Coating only the posts with cement always gives an incomplete lute.

The most reliable method to place the cement into the prepared space is with the help of a lentulo spiral as describer by D'Arcangelo $C$ et al (15), as it gives better spinning and spreading of the cement in post space, due to centrifugal dispersion of the cement. This method of spreading helps in reducing the voids and increases contact of the cement with the walls leading to optimal retention.

There are other methods of cementation that are used sometimes but have some drawbacks:-

i. Endodontic explorer- It incorporates a vertical pumping that channels the cement efficiently to the apical region but not along the lateral walls, possibly because of adherence of the cement to the instrument.

ii. Direct application- Cement is applied to the post and transported to the canal. The voids in the apical region result from the presence of air trapped as the post is seated. During final seating, the air incorporated into the cement is forced along the shaft of the post to create voids.

iii. Paper point- It creates the greatest number of voids. During cementation, the paper point becomes saturated by absorbing liquid from the body of the cement. As the cement adheres to the moist porous paper point, the continual pumping action incorporates air into the cementing medium .

\section{Conclusion}

With such a wide variety of materials, post designs and cementation techniques for the posts available for the restoration of endodontically treated teeth, the clinician should be selective in choosing the post system that best fits the individual needs of each tooth.

\section{References}

1. Stockton LW. Factors affecting retention of post systems: a literature review. J Prosthet Dent. 1999; 81: 380-5.

2. Kurer HG, Combe EC, Grant AA. Factors influencing the retention of dowels. J Prosthet Dent. 1977; 38:515-25.

3. Standlee JP, Caputo AA, Hanson EC. Retention of endodontic dowels: Effect of cement, dowel length, diameter and design. J Prosthet Dent. 1978; 39: 400-05.

4. Johnson JK, Sakumura JS. Dowel form and tensile force. J Prosthet Dent. 1978; 40: 645-9.

5. Balbosh A, Kern M. Effect of surface treatment on retention of glass-fiber endodontic posts. J Prosthet Dent. 2006; 95: 218-23.

6. Nergiz I, Schmega P, Platzer U, McMullan-Vogel CG. Effect of different surface textures on retentive strength of tapered posts. J Prosthet Dent. 1997; 78: 451-7.

7. D'Arcangelo C, D'Amario M, Vadini M, De Angelis F, Caputi S. 
Influence of surface treatments on the flexure properties of fiber posts. J. Endodon. 2007; 33: 864-7

8. Monticelli F, Ferrari M, Toledano M. Cement system and surface treatment selection for fiber post luting. Med Oral Patol Oral Cir Bucal 2008; 13(3): E214-21

9. Chan FW, Harcourt JK. The effect of post adaptation in the root canal on retention of posts cemented with various cements. Aust Dent J. 1993; 38: 39-45.

10. Mayhew JT, Windchy AM., Goldsmith LJ., Gettleman L. Effect of root canal sealers and irrigation agents on retention of preformed posts luted with resin cement. J Endod. 2000; 26(6): 341-4.

11. Keles A, Koseoglu M. Dissolution of root canal sealers in EDTA and $\mathrm{NaOCl}$ solutions. J Am Dent Assoc. 2009; 140: 74-9

12. Assif D, Ferber A. Retention of dowels using a composite resin as a cementing medium. J Prosthet Dent. 1982; 48: 292-6.

13. Monticelli F, Toledano M, Tay FR, Cury AH, Goracci C, Ferrari M. Post-surface conditioning improves interfacial adhesion in post/core restorations. Dent Mater. 2006; 22: 602-9

14. Turner CH. The retention of dental posts. J Dent. 1982; 10(2): 15465.

15. D'Arcangelo C, D'Amario M, Vadini M, De Angelis F, Caputi S. An evaluation of luting agent application technique effect on fibre post retention. J Dent. 2008; 36: 235-40 